\title{
Commentary \\ Between prediction, education, and quality control: simulation models in critical care
}

\author{
Herwig Gerlach and Susanne Toussaint
}

\begin{abstract}
Department of Anaesthesia, Intensive Care Medicine, and Pain Management, Vivantes - Klinikum Neukölln, Rudower Strasse 48, D-12313 Berlin, Germany
\end{abstract}

Corresponding author: Herwig Gerlach, herwig.gerlach@vivantes.de

Published: 6 July 2007

This article is online at http://ccforum.com/content/11/4/146

(C) 2007 BioMed Central Ltd

See related research by Saka et al., http://ccforum.com/content/11/3/R65

Critical Care 2007, 11:146 (doi:10.1186/cc5950)

\begin{abstract}
Today, computer-aided strategies in social sciences are an indispensable component of teaching programs. In recent years, microsimulation modeling has gained attention in its ability to represent predicted physiological developments visually, thus providing the user with a full understanding of the impacts of a proposed scheme. There are several microsimulation models in human medicine, and they can be either dynamic or static. If the model is dynamic the course of variables changes over time; in contrast, in the static case time constancy is assumed. In critical care there have been several approaches to implement microsimulation models to predict outcome. This commentary describes current approaches for predicting disease progression by using dynamic microsimulation in pneumonia-related sepsis.
\end{abstract}

In the previous issue of Critical Care, Saka and colleagues describe one of the latest developments of complex microsimulation [1]. In social sciences, in economy, and in technical areas, the technique of simulation is widely accepted as an important intervention that can prevent adverse events [2]. Simulation is the concept of building an environment or part of the environment resembling a real-life environment in appearance and behavior. Basically, there are three main reasons for using simulation: as a method for quality control, as a teaching tool, and for the prediction of time courses. Simulation for quality control is the domain of technical areas. In major industry, no production line, no large software, no novel technical concept would ever be possible today without previous testing with simulation programs to exclude major flaws.

In social sciences, education and prediction are the main fields of computer-aided software tools. There are two kinds of simulator for health care education: macrosimulators and microsimulators. Macrosimulators have a physical component, usually a mannequin or a body-part module, and microsimulators are purely computer-based. Both types can be further regarded as either simple or complex, depending on the complexity of the topic to be learned [3]. This results in four possible subtypes of simulator. Although the micro/ macro differentiation is discrete, the simplicity/complexity aspect is not. Simple simulators, often called part-task simulators, teach simple algorithms or procedures, involving only a few aspects of a problem. Complex simulators target more complex issues that integrate several aspects of a problem [3].

Simple microsimulators have been known since the 1960s: Kelman developed a 'digital computer subroutine' to translate a measured oxygen tension into a predicted saturation [4]. In the following 5 years, this was also practiced for carbon dioxide content [5], and ended up with the development of an artificial 'lung model' in 1970 [6]. With regard to the technical equipment available in these days, Kelman's work has to be considered a milestone of simulation, and was definitely one of the first crucial steps in the development of mathematical models for human physiology. Meanwhile, these mathematical models are further differentiated into 'forward' and 'backward' (or inverse) processes [7]. This subtyping depends on the characterization of input and output variables of the mathematical model. For a forward process, the input variables are theoretical inputs, whereas the outputs are physiological parameters, which are usually measured. The model therefore 'predicts' the outcomes. This forward process is the most common way in which biomathematical models have been used in medicine $[8,9]$. The backward processes are much more complicated: these models take measured variables such as blood gases as inputs, and seek to provide an inverse description; an example of the latter is the anatomy of the lung. In fact, computed tomography scans are based on inverse mathematical processing.

In the paper by Saka and colleagues, the investigators took data from a large cohort of 1,888 patients with severe sepsis based on community-acquired pneumonia [1]. In contrast to 
former forward models, which were developed to predict outcome in a stationary manner, namely using predefined states such as septic, severely septic, or dead [10,11], this project was aimed at simulating dynamic disease progression (or recovery) by using time-dependent inputs. In a recent paper a similar approach was chosen, also using the daily Sepsis-related Organ Failure Assessment (SOFA) score $[12,13]$. The paper by Saka and colleagues, however, included additional data from the clinical course before they entered the intensive care unit (age, gender, and ethnicity), thus 'recreating individual patients' whose entire disease course reproduced the rate of change of severity of illness. The investigators tested eight different algorithms with different sets of input variables, and the ability to predict the clinical course by some of these models is amazing, as seen in Fig. 3 of the paper [1]. Moreover, the analysis nicely demonstrates that the quality of prediction is dependent on the quality of input data: the more restrictive the criteria are, the more closely the model can predict actual experience. For instance, the 'real' data contained 1,787 discharges from the intensive care unit; the simulation algorithms predicted between 1,779 and 1,804 discharges! The best results were seen when the model also incorporated information on the duration of illness and the direction of progression.

Although the approach presented is highly data-intensive, the model demonstrates that cohort-level characteristics can be reproduced in a dynamic manner. The use of such a dynamic microsimulation cannot be foreseen, but it may have enormous potential for the future: teaching tools, feedback strategies ('briefing' and 'debriefing' after treatment), protocol development, and cost-effectiveness analysis are just a few approaches. This example of current developments of complex microsimulators may be considered as 'just a footprint', but even if we do not know what microsimulation will look like in 5 to 10 years, the direction is clear: education of health care professionals is changing markedly, and there are many more opportunities across various medical fields for expanding the use of simulations. Microsimulation in critical care is no experiment, no hobby; it is present and part of the very near future. If intensivists remain at the barrier, they will definitely miss the train!

\section{Competing interests}

The authors declare that they have no competing interests.

\section{References}

1. Saka G, Kreke JE, Schaefer AJ, Chang CCH, Roberts MS, Angus $D C$ for the GenIMS Investigators: Use of dynamic microsimulation to predict disease progression in patients with pneumonia-related sepsis. Crit Care 2007, 11:R65.

2. Maudsley GS: Science, critical thinking and competency for tomorrow's doctors: a review of terms and concepts. $J$ Med Educ 2000, 34:53-60.

3. Christensen UJ, Heffernan D, Barach P: Microsimulators in medical education: an overview. Simulation Gaming 2001, 32: 250-262.

4. Kelman GR: Digital computer subroutine for the conversion of oxygen tension into saturation. J Appl Physiol 1966, 21:13751376.
5. Kelman GR: Digital computer preocedure for the conversion of $\mathrm{P}_{\mathrm{CO} 2}$ into blood $\mathrm{CO}_{2}$ content. Respir Physiol 1967, 3:111-115.

6. Kelman GR: A new lung model: an investigation with the aid of a digital computer. Comput Biomed Res 1970, 3:241-248.

7. Hahn CEW, Farmery AD: Gas exchange modelling: no more gills, please. Br J Anaesth 2003, 91:2-15.

8. Joyce CJ, Williams AB: Kinetics of absorption atelectasis during anesthesia: a mathematical model. J Appl Physiol 1999, 86:1116-1125.

9. Peyton PJ, Robinson GJB, Thompson B: Effect of ventilationperfusion imhomogeneity and $\mathrm{N}_{2} \mathrm{O}$ on oxygenation: physiological modelling of gas exchange. J Appl Physiol 2001, 91: 17-25.

10. Bauerle R, Rucker A, Schmandra TC, Holzer K, Encke A, Hanisch E: Markov cohort simulation study reveals evidence for sexbased risk differences in intensive care unit patients. $A m ~ J$ Surg 2000, 179:207-211.

11. Rangel-Frausto MS, Pittet D, Hwang T, Woolson RF, Wenzel RP: The dynamics of disease progression in sepsis: Markov modeling describing the natural history and the likely impact of effective antisepsis agents. Crit Infect Dis 1998, 27:185-190.

12. Vincent JL, Moreno R, Takala J, Willats S, De Mendonca A, Bruining H, Reinhart K, Suter PM, Thijs LG: The SOFA (Sepsisrelated Organ Failure Assessment) score to describe organ dysfunction/failure. On behalf of the Working Group on Sepsis-related Problems of the European Society of Intensive Care Medicine. Intensive Care Med 1996, 22:707-710.

13. Clermont G, Kaplan V, Moreno R, Vincent JL, Linde-Zwirble WT Hout BV, Angus DC: Dynamic microsimulation to model multiple outcomes in cohorts of critically ill patients. Intensive Care Med 2004, 30:2237-2244. 